\title{
Plataforma flexible para nodos sensores inalámbricos orientada a la medición de vibraciones en puentes
}

\author{
A flexible platform for wireless sensor nodes intended to measure bridges vibrations
}

\author{
Javier Belmonte ${ }^{\# 1}$, Néstor Marcón ${ }^{\# 2}$, Marcelo Pistarelli ${ }^{\# 3}$, Rosa Corti ${ }^{\# 4}$, Roberto Martínez ${ }^{\# 5}$, Juan Vecchio ${ }^{\# 6}$ \\ "Ciencias Exactas, Ingeniería y Agrimensura, Universidad Nacional de Rosario, Pellegrini 250, Rosario, Argentina \\ ${ }^{1}$ belmonte, ${ }^{3}$ mpistare, ${ }^{4}$ rcorti, ${ }^{5}$ romamarefceia.unr.edu.ar \\ ${ }^{2}$ nestormarconeyahoo.com.ar \\ ${ }^{6}$ juanpivec@gmail.com
}

Recibido: 02/10/19; Aceptado: 06/01/20

\begin{abstract}
Traditional wireless sensor networks work with scalar data like temperature o humidity because they have strong restrictions on bandwidth and energy consumption. Nevertheless, in recent years, technological advances have permitted a significant growth in the use of these networks for processing and transmitting complex signals like audio, video or vibrations. This work reports the development of an adaptable platform for sensor nodes, which are able to measure, process, store and wirelessly transmit diverse physical variables. The design of these nodes allows the adaptation of them for the use in applications that require to measure and process signals of varied complexity. In addition, the nodes permit the wireless transmission of the information obtained from the local processing of the signals mentioned above. Finally, the adaptation of the platform to measure vibration in road bridges is described as a case study.
\end{abstract}

Keywords: sensor networks; civil structures; vibrations

Resumen-Las redes inalámbricas de sensores inteligentes tradicionales trabajan con datos escalares como temperatura o humedad, debido a que poseen fuertes restricciones de ancho de banda y consumo de energía. Sin embargo, en los últimos años, los avances tecnológicos han permitido un crecimiento significativo del uso de estas redes para procesamiento y transmisión de señales complejas como audio, video o vibraciones. En este trabajo se reporta el desarrollo de una plataforma flexible para nodos sensores capaces de medir, procesar, almacenar $y$ transmitir inalámbricamente distintas variables físicas. El diseño de estos nodos permite su adaptación para el uso en aplicaciones que requieran mediciones $y$ procesamiento de señales de complejidad diversa. Asimismo, los nodos permiten la transmisión inalámbrica de la información obtenida a partir del procesamiento local de las señales mencionadas. Finalmente, como caso de estudio se describe la adecuación de la plataforma para la medición de vibraciones en puentes carreteros.

Palabras clave: redes de sensores; estructuras civiles; vibraciones

\section{INTRODUCCIÓN}

Las Redes Inalámbricas de Sensores Inteligentes tradicionales (RISI) trabajan con datos escalares como temperatura, humedad o localización de objetos, debido a que poseen fuertes restricciones de ancho de banda y consumo de energía [1]. Sin embargo, los incrementos constantes en las escalas de integración, la aparición de protocolos de la más diversa índole, el aumento en la eficiencia de las baterías utilizadas en estas redes, las mejoras alcanzadas en los sensores y dispositivos de ultrabajo consumo, han permitido su evolución para poder incorporarlas en dominios que incluyen variables de mayor complejidad [2]. En forma paralela a esta evolución se produce una explosión de los contenidos multimedia y sus técnicas de adquisición, procesamiento y transmisión [3]. Pese a los grandes requerimientos de energía y ancho de banda normalmente necesarios para este tipo de datos, contrarios a las premisas de diseño de las RISI, en los últimos años han aparecido trabajos y tecnologías tendientes a definir un puente entre ambos universos, dando origen a las Redes Inalámbricas de Sensores Multimedia (RISM). Una RISM se define como una red de dispositivos inalámbricos que permiten la adquisición y transmisión de flujos de video, audio o imágenes, sumado al manejo de señales escalares habituales de las RISI [4]. En este sentido, se han llevado a cabo numerosos esfuerzos tendientes principalmente a adaptar el uso de la red para el transporte de estos flujos [5].

Es importante destacar, que en muchas aplicaciones no resulta de utilidad la transmisión de las señales complejas en sí mismas, sino datos extraídos u obtenidos de ellas en función de lo que efectivamente se requiera adquirir o medir. Incluso, aprovechar la naturaleza distribuida de las RISI para procesar los datos en los nodos sensores que realizan las mediciones, eliminar redundancias en la información y optimizar las transmisiones [6]. Este último enfoque, ha sido utilizado para abordar el desarrollo de aplicaciones en el dominio de la supervisión ambiental, sobre la base de técnicas de recolección y procesamiento local/distribuido para reducir en forma considerable la circulación de información en el sistema [7]. 
Un aspecto a considerar en esta área de trabajo es la dificultad existente para la definición de requerimientos típicos de las redes de sensores, debido a que se utilizan en aplicaciones y dominios extremadamente variados [8]. Los nodos sensores que las componen se deben modificar o, incluso, rediseñar atendiendo a factores particulares de cada aplicación y a las condiciones del ambiente donde se los emplaza [9]. Por lo tanto, la implementación final de estos dispositivos incluye, en la mayoría de los casos, componentes específicos para cubrir los requerimientos del problema, con el fin de obtener una solución eficiente que prolongue el tiempo de vida del sistema [10]. Sin embargo, esta situación impacta negativamente en términos de complejidad, costo y tiempo de desarrollo de las aplicaciones [9]. En los últimos años, se han propuesto diversas plataformas que se inscriben en dos grandes enfoques, las que priorizan el bajo consumo y restringen los recursos a los específicos de la aplicación y las que ofrecen una mayor generalidad de uso a costa de un mayor consumo [11]. Esta situación fue considerada por los autores de este artículo desde el inicio del desarrollo, proponiendo el diseño de una plataforma flexible que se pueda integrar en una RISI para su uso en diversas aplicaciones. Este enfoque jugó un rol fundamental en la selección de los componentes que se incluyen en la versión base de la plataforma y en las posibilidades de expansión y adaptación para otras versiones orientadas a aplicaciones que reúnan un conjunto de requerimientos comunes.

El resto del trabajo se organiza según se indica a continuación. La sección II plantea la problemática de la supervisión del estado de las estructuras civiles, la sección III describe la plataforma flexible, cuya orientación para el caso de estudio elegido se presenta en la sección IV. Finalmente, en la sección $\mathrm{V}$ se resumen las conclusiones obtenidas y futuros trabajos.

\section{SUPERVISIÓN DE LA SALUD ESTRUCTURAL EN CONSTRUCCIONES CIVILES}

Las RISI tienen entre sus numerosos campos de aplicación la supervisión de la salud estructural en construcciones civiles (SHM - Structural Health Monitoring). SHM es un proceso que consiste en supervisar en forma continua la respuesta de las estructuras para definir su estado y prevenir accidentes. Para ello es necesario recolectar datos adquiridos por sensores, emplazados en diversas locaciones de la estructura, en períodos de tiempo que pueden ser de corto o largo plazo dependiendo del estudio que se realice [12]. Las variables de interés en estas aplicaciones son variadas, pero entre ellas se pueden mencionar factores ambientales, como temperatura y humedad. Asimismo, resultan de interés valores de aceleración y velocidad sobre las estructuras.

Tradicionalmente, los sistemas SHM incluían redes de sensores cableadas pero, la alta confiabilidad y los bajos costos de instalación y mantenimiento de las redes inalámbricas las han vuelto muy populares en este dominio de trabajo [13]. Las RISI, que han abaratado sensiblemente la instalación de un SHM, han incrementado el uso de estos sistemas y son relevantes en la tarea de extender el tiempo de vida útil de muchas construcciones, al contribuir a la detección temprana de daño estructural. En este sentido, se han desplegado en numerosas construcciones críticas como presas, puentes, edificios de gran altura, estadios y también en vehículos aéreos y barcos, contribuyendo a la seguridad del público en general [14].

Los puentes para tránsito vehicular se han vuelto muy habituales tanto en las rutas como en las ciudades y, como consecuencia del crecimiento de los sistemas de transporte de cargas, sufren riesgos de daño estructural a medida que transcurre el tiempo. Los métodos estándar de evaluación del estado de estas estructuras consisten en inspecciones visuales periódicas que suelen ser infrecuentes y conllevan un importante grado de subjetividad [15]. Es así, que las RISI se han vuelto una herramienta de gran utilidad para evaluar la salud estructural de distintos tipos de puentes, permitiendo acceder a mediciones confiables en forma sistemática [15].

Existen diversas pruebas dinámicas que se utilizan para analizar el estado de los puentes carreteros. En particular, los ensayos de vibraciones son estudios no destructivos que brindan información a los especialistas para determinar características del puente, tales como frecuencias naturales de vibración, forma modal y amortiguamiento [16]. En este marco presentamos, como caso de estudio, una versión de la plataforma flexible para nodos sensores, que se presentará en la sección III, orientada a la medición de vibraciones en puentes carreteros.

\section{DISEÑO DE LA PLATAFORMA}

\section{A. Especificaciones generales}

El modo de trabajo del nodo sensor consiste en la realización de mediciones de variables del medio, que se procesan y almacenan localmente. Periódicamente, estos datos son requeridos y descargados por un dispositivo de telefonía móvil. Este último proceso se aborda combinando dos protocolos de comunicación inalámbrica, Bluetooth de baja energía (BLE) y WiFi, con el objetivo de reducir el consumo de energía. El modo de trabajo elegido y el requerimiento de flexibilidad para la plataforma de los nodos sensores, se encaró diseñando una configuración base, capaz de cumplir con las siguientes especificaciones:

- Autonomía de batería para asegurar su funcionamiento por un mínimo de 30 días.

- Estanqueidad del dispositivo para poder ser emplazado en exteriores.

- Capacidad de procesamiento y almacenamiento local de datos.

- Acceso remoto de los datos almacenados localmente.

- Posibilidad de medición de magnitudes físicas diversas, soportando los correspondientes protocolos de comunicación on board de los dispositivos.

El diseño propuesto se puede personalizar para las aplicaciones en las que se lo utilice, a partir de la incorporación de componentes específicos necesarios para cada caso. Las distintas versiones de la plataforma base, se logran en virtud de la flexibilidad que le otorgan las características que se eligieron para su diseño. Se incluye dentro de esta flexibilidad la posibilidad de adquisición, procesamiento local y transmisión en tiempo real de las mediciones para los casos que esta modalidad sea requerida. 


\section{B. Características del Hardware}

En el diseño y selección de los componentes se priorizó el ahorro de energía a fin de prolongar la vida útil del nodo cuya fuente principal de energía son baterías, como es habitual en las redes de sensores. Sin embargo, el diseño de la fuente de alimentación contempló la posibilidad de incluir dispositivos sensores de consumo elevado y también la provisión de energía externa permanente o semipermanente, para, de esta manera, dar versatilidad a la provisión de energía de la plataforma para abordar aplicaciones de características diversas. Esto se logró basando el diseño en un regulador que alterna entre dos modos de trabajo: PWM (Pulse Width Modulation), cuando la operación de la plataforma presenta cargas elevadas y PFM (Pulse Frequency Modulation), cuando la carga es baja como cuando la plataforma opera en alguno de los estadios de ahorro de energía. Adicionalmente, el uso de baterías de Li-ion y la disponibilidad de un cargador integrado permiten contemplar todos los escenarios posibles para la disponibilidad de energía.

Para el núcleo del desarrollo se seleccionó el módulo EMW3239 de la empresa MXCHIP debido a que integra un microcontrolador ARM de 32 bits, un chip WIFI/BLE y una memoria flash de $2 \mathrm{MB}$. La Fig. 1 presenta el diagrama en bloques del módulo mencionado.

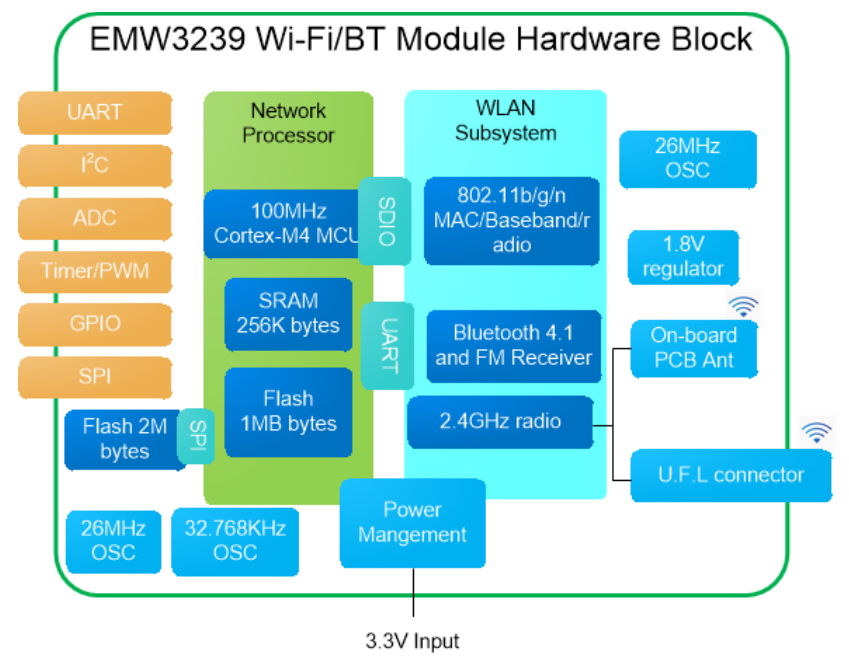

Fig. 1. Diagrama en bloques del módulo EMW3239

La plataforma diseñada cumple con las especificaciones anteriormente definidas a partir de las siguientes características:

- Disponibilidad de módulos de comunicaciones I2C, SPI y UART, que permiten conectar distintos tipos de sensores para cubrir requerimientos de diversas aplicaciones.

- Capacidad de procesamiento para soportar un protocolo de mayor nivel. El equipo de trabajo ha desarrollado CLUDITEM, un protocolo de encaminamiento jerárquico orientado a las aplicaciones de supervisión ambiental, que podrá ser incorporado a esta plataforma [7], [17].

- Disponibilidad de cálculo en punto flotante y memoria RAM para reducir la grabación de datos a la FLASH externa y posibilitar el procesamiento local de datos de un nodo o cluster [17].
- Disponibilidad de WiFi y BLE y posibilidad de agregar otros protocolos, como el 802.15.4 que brinda soporte a CLUDITEM y módulos GPRS.

- Modos de bajo consumo del controlador de WiFi/BLE para cumplir con la autonomía de funcionamiento requerida.

- Flexibilidad en el manejo de energía con eficiente administración para situaciones tanto de alta como de baja carga.

\section{Características del Software}

Distinguimos aquí entre el firmware, que corre y da funcionalidad al nodo propiamente dicho, y una aplicación destinada a correr en un teléfono celular bajo Android encargada de brindar facilidades para la configuración de nodo y la recuperación de los datos adquiridos.

\section{1) Firmware}

El firmware incluye la implementación de los servicios esenciales de administración del consumo, comunicaciones, datalogging, y modos de acceso para transferencia de la información registrada.

El diseño contempla una fácil y rápida adaptación de estos módulos, así como la incorporación de otros específicos a la versión que se defina para la plataforma. El desarrollo corre bajo FreeRTOS y contempla la definición de distintos threads para implementar las tareas de adquisición de datos y transmisión de los mismos.

El firmware inicializa pines y periféricos del micro, configura la memoria externa y los sensores y crea los threads de adquisición y almacenamiento (o transmisión en tiempo real si correspondiera). También configura por defecto la conexión BLE y, por último, pone la plataforma en el modo de bajo consumo de energía. De este estado sale bajo dos circunstancias: por interrupción de algún sensor en la adquisición o para hacer un anuncio BLE en busca de la aplicación para descarga de información. El registro de una función de callback al servidor GATT (Generic Attribute Profile) para el manejo de eventos BLE de la plataforma, sumada a la emisión periódica de anuncios de conexión a la espera de ser escaneado por la aplicación, completan los trabajos realizados bajo BLE. El proceso de adquisición de datos, a los fines de ahorrar energía, almacena las mediciones en un buffer interno y, sólo cuando éste se llena, los transfiere a la memoria externa. Finalmente, una tarea específica se ejecuta, a partir del evento BLE de solicitud de transferencia, a los fines de transmitir mediante sockets y vía WiFi los datos almacenados en la plataforma al celular destino. El uso de WiFi, con acceso local autenticado mediante credenciales apropiadas (SSID / usuario / contraseña) bajo WPA2-AES Personal, brinda un nivel de seguridad compatible con las aplicaciones de la plataforma. Una vez terminada la transferencia, se retorna la emisión periódica de anuncios BLE.

\section{2) Aplicación}

El acceso a las mediciones almacenadas en el nodo se realiza mediante la aplicación Android que se instala en el dispositivo móvil del usuario e interactúa con el firmware a partir de responder a alguno de los anuncios periódicos que realiza el mismo. Si bien el modo de trabajo para las transferencias será prácticamente invariable aún para un amplio espectro de uso de la plataforma, se prevé un 
versionado de la misma a los efectos de realizar funciones de configuración del nodo evitando así modificaciones del firmware ante cambios de escenarios de adquisición. Modificaciones similares de la aplicación son esperables para escenarios de transmisión de la información en tiempo real.

\section{CASO DE ESTUDIO: MEDICIÓN DE VIBRACIONES EN PUENTES CARRETEROS}

Los puentes carreteros, son estructuras civiles que se deterioran con el paso del tiempo como consecuencia de varios factores, entre ellos las vibraciones producidas por el tránsito vehicular. Para obtener los datos necesarios para estos estudios, es habitual que se utilicen acelerómetros triaxiales incorporados a los nodos sensores que se emplazan en los puentes. Los datos referidos a estas vibraciones y su posterior análisis permiten evaluar el efecto sobre las estructuras. Este caso de estudio, una versión de la plataforma flexible presentada en este trabajo, está en etapa de desarrollo en un proyecto de fin de carrera.

Para este desarrollo se contó con el asesoramiento de investigadores del Instituto de Mecánica Aplicada y Estructuras (IMAE) de la Facultad de Ciencias Exactas, Ingeniería y Agrimensura de la Universidad Nacional de Rosario. El caso de estudio tiene por fin medir, procesar, almacenar y transmitir en forma inalámbrica variables físicas de interés, en particular vibraciones obtenidas en distintos puntos del puente. Los datos se recolectan en forma periódica off line, para lo cual se utilizan las capacidades de datalogging de la plataforma, por un lado, y la flexibilidad en sus modos de comunicación, por otro. El nodo sensor debe hacerse solidario al puente bajo estudio, por lo que las condiciones de montaje y estanqueidad del diseño son imprescindibles. Respecto de la medición de vibraciones en la estructura, estudios preliminares muestran que estarán comprendidas en el orden de milésimas de la aceleración de la gravedad (entre $0,02 \mathrm{~m} / \mathrm{s}^{2}$ y $0,3 \mathrm{~m} / \mathrm{s}^{2}$ ) con un ancho de banda $25 \mathrm{~Hz}$ como máximo.

\section{A. Versión de la plataforma para el caso de estudio}

Para este caso de estudio, el versionado del nodo incluyó un acelerómetro triaxial de 16 bits (LIS3DSH), cuyas prestaciones son suficientes para cumplir con los requerimientos establecidos en los estudios preliminares antes mencionados.

En adición a la memoria base de $2 \mathrm{MB}$ de la plataforma, se agregó en esta versión una memoria SPI de 500MB a los fines de extender sustancialmente la capacidad de almacenamiento del nodo y posibilitar la recolección y almacenamiento de datos durante 30 días.

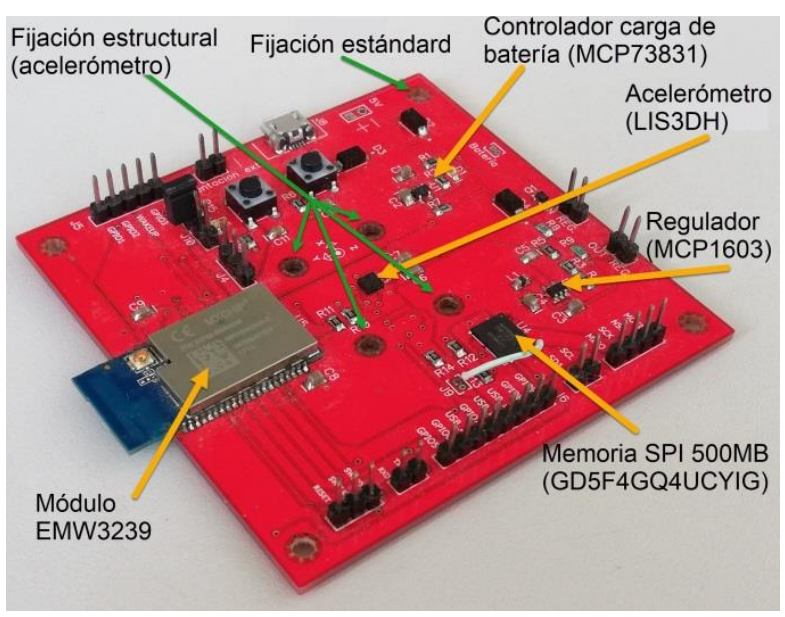

Fig. 2. Plataforma versionada para el caso de estudio

Existen otras plataformas para aplicaciones en salud estructural de construcciones civiles. La Tabla I, presenta características de prototipos académicos obtenidos a partir de información publicada por sus autores. Los datos consignados corresponden a desarrollos académicos de los últimos cuatro años, que se reportan en [18], a los que se han agregado las características correspondientes al prototipo aquí presentado. Como puede apreciarse, las características y prestaciones del nodo basado en la

TABLA I

CARACTERÍSTICAS DE PLATAFORMAS PARA NODOS SENSORES PARA SUPERVISIÓN DE SALUD ESTRUCTURAL EN CONSTRUCCIONES CIVILES

\begin{tabular}{|c|c|c|c|c|c|c|}
\hline $\begin{array}{l}\text { Prototipo } \\
\text { Año }\end{array}$ & Ensayos & $\begin{array}{c}\text { Resolución } \\
\text { A/D }\end{array}$ & Procesador & Memoria & Comunicación & Rango de datos \\
\hline $\begin{array}{l}\text { PWSMS } \\
2016 \\
\end{array}$ & $\begin{array}{l}\text { Quayside } \\
\text { cranes }\end{array}$ & - & TI MSP430F149 & $\begin{array}{c}2 \text { KB SRAM + } 60 \mathrm{~KB} \\
\text { Flash }\end{array}$ & IEEE802.15.4 & $250 \mathrm{Kbps}$ \\
\hline $\begin{array}{l}\text { Prototipo } \\
2016\end{array}$ & Laboratorio & 12 bits & $\begin{array}{c}\text { ARM Cortex M3 } \\
\text { EFM 32G222F128 }\end{array}$ & $\begin{array}{c}16 \text { KB SRAM + } 128 \\
\text { KB Flash }\end{array}$ & IEEE802.15.4 & $\begin{array}{c}250 \mathrm{Kbps} / 100 \\
\mathrm{~m}\end{array}$ \\
\hline $\begin{array}{c}\text { Active } \\
\text { Wireless } \\
\text { System } \\
2017\end{array}$ & Laboratorio & 10 bits & ATmega644PA & $\begin{array}{c}4 \text { KB SRAM + } 64 \text { KB } \\
\text { Flash }\end{array}$ & IEEE802.15.4 & $\begin{array}{c}250 \mathrm{Kbps} / 1,2 \\
\mathrm{~km}\end{array}$ \\
\hline $\begin{array}{c}\text { Wireless } \\
\text { AE - } 2017\end{array}$ & Laboratorio & 12 bits & $\begin{array}{c}\text { ARM Cortex M4 } \\
\text { STM32F407 V GT6 }\end{array}$ & $192+4$ KB SRAM & $\begin{array}{l}\text { IEEE802.11 } \\
\mathrm{b} / \mathrm{g} / \mathrm{n}\end{array}$ & $6 \mathrm{Mbps}$ \\
\hline $\begin{array}{l}\text { Prototipo } \\
2017 \\
\end{array}$ & Puente & 10 bits & Microchip PIC18 & $\begin{array}{c}4 \mathrm{~KB} \text { RAM + } 128 \mathrm{~KB} \\
\text { Flash }\end{array}$ & $2.4 \mathrm{GHz}$ & $500 \mathrm{Kbps}$ \\
\hline $\begin{array}{c}\text { Xnode } \\
2018 \\
\end{array}$ & Laboratorio & 24 bits & NXP LPC4357 & 32 MB SDRAM & $\begin{array}{l}\text { IEEE802.15.4, } \\
\text { 6LoWPAN }\end{array}$ & $\begin{array}{c}250 \mathrm{Kbps} / 1 \\
\mathrm{~km}\end{array}$ \\
\hline $\begin{array}{c}\text { WSHMS } \\
2019\end{array}$ & Laboratorio & 12 bits & STM32L476ZE & $\begin{array}{c}128 \text { KB SRAM + } 1 \mathrm{MB} \\
\text { Flash }\end{array}$ & IEEE802.15.4 & $\begin{array}{c}250 \mathrm{Kbps} / \\
100 \mathrm{~m}\end{array}$ \\
\hline $\begin{array}{l}\text { Prototipo } \\
2019(*)\end{array}$ & Laboratorio & 16 bits & ARM Cortex M4 & $\begin{array}{c}256 \mathrm{~KB} \text { SRAM + } 2 \mathrm{MB} \\
\text { FLASH + 500 MB } \\
\text { FLASH (externa) }\end{array}$ & $\mathrm{BLE} / \mathrm{WiFi}$ & $6 \mathrm{Mbps} / 100 \mathrm{~m}$ \\
\hline
\end{tabular}

(*) Prototipo presentado en este trabajo 
plataforma flexible, que se describe en este artículo, son comparables y, en algunos casos, exceden a las exhibidas por los otros desarrollos citados. A esto se agrega la versatilidad de la plataforma base, según se consigna en sección III, que facilita su utilización futura en otros dominios de aplicación.

\section{B. Software}

Los nodos sensores que se emplazan en el puente carretero, respondiendo a los requerimientos de los expertos, son responsables de dos tareas:

- Adquirir y almacenar localmente las muestras de aceleración en los tres ejes cuyos valores superan un umbral definido.

- Transmitir la información almacenada hacia un teléfono móvil, vía WiFi, cuando el usuario así lo solicite.

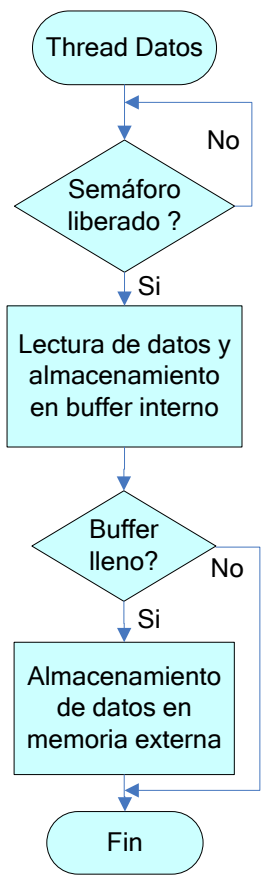

Fig. 3. Thread de adquisición de datos

El funcionamiento general de la plataforma flexible, orientada al caso de estudio elegido, responde al esquema de trabajo general descripto en el apartado II. C. 1). Esto es, el usuario configura los parámetros asociados con la adquisición de datos, en este caso, frecuencia de muestreo, escala, filtro analógico pasa bajos y umbral para selección de mediciones, por medio de la aplicación instalada en su celular y a continuación se realiza la inicialización general de la plataforma. A partir de ese momento los nodos registran la información de interés, que se transmite cada vez que el usuario lo solicite.

La tarea de registro en memoria de los datos adquiridos por el acelerómetro, se implementó mediante el thread que se ejecuta cuando dicho sensor emite una interrupción. La Fig. 3 muestra la utilización del buffer interno en RAM y la grabación final en memoria, con el objetivo de disminuir el consumo de energía.

La tarea de envío de mediciones desde la plataforma hacia el celular del usuario se implementa en forma estándar combinando los protocolos BLE y WiFi. La respuesta a uno de los avisos periódicos vía BLE, desactiva los mismos y se activa la comunicación $\mathrm{WiFi}$, responsable de la transmisión efectiva de los datos almacenados localmente. Al finalizar la transmisión se apaga $\mathrm{WiFi}$ y se reanuda el envío periódico de anuncios BLE. La Fig. 4, que resume el thread WiFi, ilustra el proceso descripto.

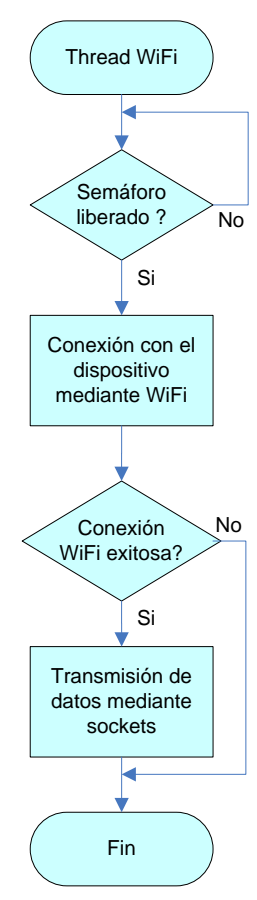

Fig. 4. Thread WiFi

\section{Ensayos de laboratorio}

Se realizaron ensayos preliminares de funcionamiento del nodo sensor presentado en este trabajo, en el Laboratorio de Tecnología de Materiales del IMAE. Para ello se compararon los datos adquiridos por un equipo de medición de vibraciones proporcionado por el Laboratorio con el nodo bajo prueba que se utilizó como patrón. Este equipo dispone de un acelerómetro de alta precisión (DYTRAN 7503D).

Los ensayos se realizaron considerando la presencia de vibraciones en un rango de frecuencias entre 0 y $50 \mathrm{~Hz}$ con una amplitud máxima de $10 \mathrm{~m} / \mathrm{s}^{2}$. El nodo sensor se configuró con un umbral de valor cero para detectar todas las vibraciones presentes, que se muestrearon con una frecuencia de $100 \mathrm{~Hz}$. La estructura sometida a vibración consistió en una viga de madera sujeta por sus extremos y ambos sensores se ubicaron en el punto medio de la viga mencionada. La fuerza excitadora consistió en impactos realizados sobre la viga en la dirección del eje vertical.

En las Fig. 5 y 6 se presentan las aceleraciones en los dominios de tiempo y frecuencia en el eje vertical medidas por el equipo patrón y por el nodo sensor.

Las gráficas de las mediciones del equipo patrón y el nodo sensor muestran similitud, tanto en el dominio de tiempo como en el de frecuencias. Esta valoración cualitativa se complementó con un análisis de regresión lineal de los datos en el dominio temporal. El mismo arrojó un factor de correlación de 0,723 . 


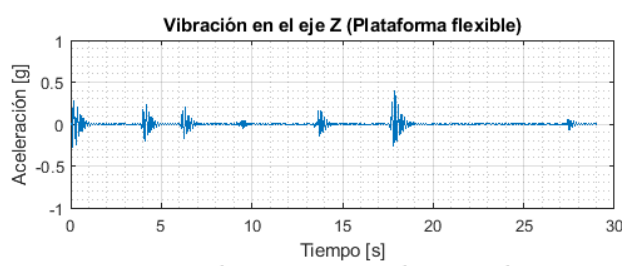

Vibración en el eje Z (Acelerómetro patrón)

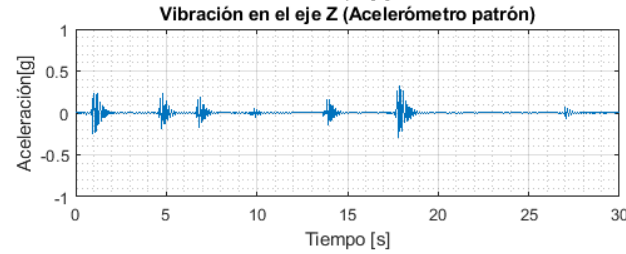

Fig. 5. Valores de vibraciones en el eje vertical en el dominio del tiempo
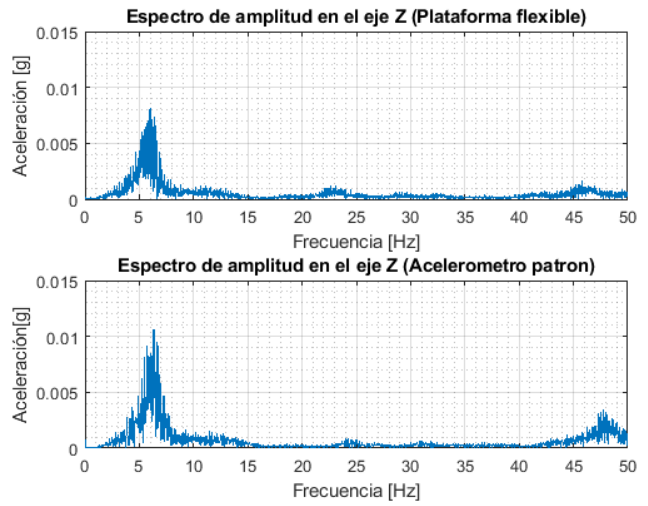

Fig. 6. Valores de vibraciones en el eje vertical en el dominio de las frecuencias

Luego de la adquisición de datos en el nodo sensor se solicitó, desde el celular destino, la descarga vía WiFi. Este ensayo se realizó a $80 \mathrm{~m}$ de distancia con línea de vista lográndose una velocidad efectiva de transferencia por TCP de 5 Mbps.

\section{CONCLUSIONES Y TRABAJO FUTURO}

En este trabajo se presentó una plataforma flexible para nodos sensores inalámbricos. El prototipo implementado no pretende ser un producto final de uso comercial sino una implementación flexible a la hora de proceder fácilmente a realizar reconfiguraciones y pruebas, aunque funcional en todos sus aspectos esenciales y a los fines perseguidos. No obstante, una versión final y estándar de la plataforma con fines comerciales puede pensarse como trabajo futuro a través de un rediseño del hardware a los fines de mejorar la robustez del mismo, como la utilización de conectores con fijaciones cautivas, o la incorporación de facilidades de actualización del firmware en forma inalámbrica (OTA), como posibles ejemplos.

Se procedió al versionado e implementación de la plataforma para el caso de estudio concreto de medición de vibraciones en puentes carreteros. Asimismo, se realizaron pruebas de funcionamiento del prototipo versionado en laboratorio. El nodo sensor cumplió con las fases de configuración, medición, registro local y transmisión de datos previstas. Resta realizar ensayos referidos a la duración de la batería y capacidad de almacenamiento, que se deberán diseñar teniendo en cuenta la densidad y tipo de tránsito vehicular. También se deberá considerar el umbral de las vibraciones a registrar y la frecuencia de recolección de datos.
El acelerómetro utilizado en esta versión de la plataforma es adecuado para las especificaciones definidas y cumple con el presupuesto disponible. Sin embargo, si alguna aplicación requiriera aumentar el factor de correlación con el equipo patrón, esto se lograría mejorando las prestaciones del acelerómetro usado.

La siguiente etapa de trabajo es el emplazamiento de una red de quince nodos sensores de esta versión, en un puente carretero en el área de la ciudad de Rosario, considerando la pertinencia de implementar el protocolo CLUDITEM y agregar procesamiento local de las mediciones almacenadas. Asimismo, se prevé extender su utilización para la adquisición de datos en otras estructuras civiles de características diferentes. Estas experiencias, así como otras proyectadas en el dominio de la supervisión ambiental, control de lagunas de estabilización y medición de calidad de aire en granjas porcinas en confinamiento, permitirán perfeccionar el diseño y validarlo, como una extensión de los objetivos planteados en este trabajo.

\section{REFERENCIAS}

[1] M. Bhende, S. J. Wagh and A. Utpat, "A Quick Survey On Wireless Sensor Networks", in Proc. Fourth International Conference on Communication Systems and Network Technologies, March 2014, pp. 160-167.

[2] F. Al-Turjman and A. Radwan, "Data Delivery in Wireless Multimedia Sensor Networks: Challenging and Defying in the IoT Era", IEEE Wireless Communications, vol. 24, Issue 5, pp. 125-131, 2017.

[3] H. Shen and G. Bai, "Routing in Wireless Multimedia Sensor Networks: A Survey and Challenges Ahead", Journal of Network and Computer Applications, vol. 71, Issue7, pp. 30-49, 2016.

[4] I.T. Almalkawi, M. Guerrero Zapata, J. N. Al-Karaki and J. MorilloPozo, "Wireless Multimedia Sensor Networks: Current Trends and Future Directions", Sensors Journal, vol. 10, pp. 6662-6717, 2010.

[5] G. Viscardi, M. Roveri, R. Camplani and C. Alippi, "Netbrick: A high-performance, low-power hardware platform for wireless and hybrid sensor networks", in Proc. IEEE 9th International Conference on Mobile Ad-Hoc and Sensor System, 2012, pp. 111117.

[6] M. Anupama Reddy, D. Anjaneyulu, D. Prasanth Varma and G. Rama Rao, "WSN in IOT Environment Interfacing with Smart Sensors using Arm7 with Zigbee for Industries", International Journal of Engineering Research and Development, vol. 12, Issue 8, pp. 54-60, 2016.

[7] R. Corti, E. Giandoménico, J. Belmonte y R. Martínez, "Implementación de una Red de Sensores para aplicaciones de supervisión ambiental", Memorias VII Jornada de Ciencia y Tecnología de SECyT/UNR, 2013, pp. 295-299.

[8] G. A. Al-Suhail, J. Mehdi and G. Nikolakopoulos. "A Practical Survey on Wireless Sensor Network Platforms", Journal of Communications Technology, Electronics and Computer Science, Issue 13, 2017.

[9] M. T. Lazarescu, "Design of a WSN Platform for Long-Term Environmental Monitoring for IoT Applications," IEEE Journal on Emerging and Selected Topics in Circuits and Systems, vol. 3, pp. 45-54, March 2013.

[10] S. Bader, M. Krämer and B. Oelmann, "A Domain-Specific Platform for Research in Environmental Wireless Sensor Networks", in Proc. of The Seventh International Conference on Sensor Technologies and Applications , 2013, pp 200 -207.

[11] J. Chen, P. Li, G. Song, Y. Tan, Y. Zheng and Y Han, "Systematic Development of a Wireless Sensor Network for Piezo-Based Sensing", Journal of Sensors, vol. 2018, pp. 1-12, 2018.

[12] K Sreevallabhan, B Nikhil Chand and S. Ramasamy, "Structural health monitoring using wireless sensor networks", in Proc IOP Conference Series: Materials Science and Engineering, vol. 263, May 2017, pp. 1-5.

[13] C. Ayyildiz, H. Erdem, T. Dirikgil, O. Dugenci, T. Kocak, F. Altun and V. Cagri Gungor. "Structure health monitoring using wireless 
Revista elektron, Vol. 4, No. 1, pp. 14-20 (2020)

sensor networks on structural elements", Ad Hoc Networks, vol. 82, pp. $68-76,2019$.

[14] A. B. Noel, A. Abdaoui, T. Elfouly, M. Hossam Ahmed, A. Badawy, and M. S. Shehata, "Structural Health Monitoring using Wireless Sensor Networks: A Comprehensive Survey", IEEE Communications Surveys \& Tutorials, vol. 19, pp 1403-1423, April 2017.

[15] T. J. Matarazzo , P. Santi, S. Pakzad, K. Carier, C. Ratti, B. Moaveni, C. Osgood, and N. Jacob, "Crowdsensing Framework for Monitoring Bridge Vibrations Using Moving Smartphones", in Proc. IEEE, vol. 106, No. 4, April 2018, pp.577 - 593.

[16] F. Navarro-Henríquez and D. Ureña-Muñoz, "Mediciones y análisis de vibraciones en el puente Virilla, ruta nacional $\mathrm{N}^{\circ} 1$ ", Tecnología en Marcha,. vol. 28, pp. 16-32, Abril-Junio 2015.

[17] R. Corti, "Clustering dinámico para tiempo de encendido mínimo en redes inalémbricas de sensores (CLUDITEM)", Tesis de Maestría en
Ingeniería de Software, Universidad Nacional de La Plata, Argentina, 2012.

[18] M. Abdulkarem, K. Samsudin, F. Z. Rokhani and M. F.Rasid, "Wireless sensor network for structural health monitoring: A contemporary review of technologies, challenges, and future direction", Structural Health Monitoring, pp. 1-43, 2019. 